\title{
The Ulam's types stability of non-linear Volterra integro- delay dynamic system with simple non-instantaneous im- pulses on time scales
}

\author{
Syed Omar Shah ${ }^{\mathrm{a}, *}$, Akbar Zada $^{\mathrm{a}}$, Cemil Tunç \\ a Department of Mathematics, University of Peshawar, Peshawar 25000, Pakistan. \\ ${ }^{b}$ Department of Mathematics, Faculty of Sciences, Yuzuncu Yil University, 65080 Van, Turkey.
}

\begin{abstract}
This manuscript presents Hyers-Ulam stability and Hyers-Ulam-Rassias stability results of non-linear Volterra integro-delay dynamic system on time scales with non-instantaneous impulses. Picard fixed point theorem is used for obtaining existence and uniqueness of solutions. By means of abstract Grönwall lemma, Grönwall's inequality on time scales and applications of Grönwall's inequality on time scales, we establish Hyers-Ulam stability and Hyers-Ulam-Rassias stability results. There are some primary lemmas, inequalities and relevant assumptions that helps in our stability results.
\end{abstract}

Keywords: Hyers-Ulam stability, time scale, impulses, delay dynamic equation, Grönwall's inequality, abstract Grönwall lemma, Banach fixed point theorem.

2010 MSC: 34N05, 34G20, 34A37, 45J05, 45M10.

(C)2019 All rights reserved.

\section{Introduction}

In 1940, in a talk before the mathematics club at the university of Wisconsin, Ulam [33, 34] presented a famed question related to the stability of homomorphisms: "When an approximate homomorphism from a group $\mathrm{G}_{1}$ to a metric group $\mathrm{G}_{2}$ can be approximated by an exact homomorphism?".

This question was answered by Hyers [13] for the case when $G_{1}$ and $G_{2}$ are assumed to be Banach spaces by using direct method. So this interesting stability, initiated by Ulam and Hyers, is called HyersUlam stability. In 1978, Rassias [25] extended Hyers-Ulam stability concept by introducing new function variables and after that it famed for the Hyers-Ulam-Rassias stability. In fact, the most interesting result was of Rassias [25] that weakens the condition for the bound of the norm of Cauchy difference $f(x+y)-$ $f(x)-f(y)$. For further details and discussions, we recommend the book by Jung [15].

At the end of 19th century, a large number of researchers contributed to the stability idea of Ulam's type for various types of differential equations. There are many advantages of Ulam's type stability in

\footnotetext{
${ }^{*}$ Corresponding author

Email addresses: omarshah89@yahoo.com, omarshahstd@uop.edu.pk (Syed Omar Shah), zadababo@yahoo.com, akbarzada@uop.edu.pk (Akbar Zada), cemtunc@yahoo.com (Cemil Tunç)
}

doi: $10.22436 / \mathrm{mns} .04 .01 .02$

Received: 2018-09-24 Revised: 2019-03-26 Accepted: 2019-05-04 
tackling problems, related to optimization techniques, numerical analysis, control theory and many more, in such situations to get an exact solution is challenging. For more details on Hyers-Ulam stability, see $[2,3,14,16-18,22,23,28-32,35,38,41-54]$.

There are several implications of simple differential equations. Anyhow, the circumstances rather change when a real world process undergoes with unexpected variations, like significant mechanical processes, blood flows, heart beats, changes in population, radio physics, pharmacokinetics, mathematical economy, chemical technology, electrical technology, chemistry, different engineering fields, control theory and so on, see $[6,7,21]$. Sufficiently many mathematical problems in such circumstances generate a polished differential equation, which is known as impulsive differential equation.

More precisely, there are three parts of differential equations with impulse impact: an instantaneous impulsive differential equation [38], in which the impulse action is defined at certain discrete points; non-instantaneous impulsive differential equation [47], it establishes the effect of impulse on an interval; and the third one is an impulse rule, in which we define a distinct and well defined collection of impulse events having an active impulse equation.

Fractional differential and integral equations [47] play a key role not only in mathematics but also in the modeling of various physical phenomena in physics, control systems and dynamical systems. In fact, fractional order derivatives and integrals are assumed to be more realistic and practical than derivatives and integrals of integral order. These are excellent tools to model genetic transformation and memory retention qualities of several systems and products.

It is to be noted that, the pioneer of the Ulam's type stability for impulsive ordinary differentiable equation is Wang et al. [36]. Following their own work, in 2014, they proved the Hyers-Ulam-Rassias stability and generalized Hyers-Ulam-Rassias stability of impulsive evolution equations on a compact interval [37] which then they extended for infinite impulses in the same paper. Wang and Zhang [39], initially offered nonlinear differential equations having fractional integrable impulses, which are more interesting. They presented four Bielecki-Ulam's type stabilities for this class of differential equations. Also Lin et al. [19] discussed the existence and stability results for impulsive integro-differential equations. The work of Wang et al. [39] was extended by Zada et al. [43] in which they discussed HyersUlam stability of higher-order nonlinear differential equations with fractional integrable impulses. They established Bielecki-Ulam-Hyers-Rassias stability, generalized Bielecki-Ulam-Hyers-Rassias stability and Bielecki-Ulam-Hyers stability for this class of differential equations on a compact interval.

However, despite the situations where only impulsive factor is involved or delay effects happened, we have a wide variety of evolutionary processes together delay and impulsive effects exist in their state. To model such phenomena which are subject to impulsive perturbations as the time delays, an impulsive delay differential equation is used.

The theory of dynamic equations on time scales has been rising fast and has acknowledged a lot of interest in recent years. This theory was introduced by Hilger [12] in 1988, with the inspiration to provide a unification of continuous and discrete calculus. For more details on time scales, see $[1,4,5,8-$ 11, 20, 24, 27, 29-31, 40, 48, 49].

Recently, Zada et al. [49] obtained very interesting results about the Hyers-Ulam stability of nonlinear impulsive Volterra integro-delay dynamic system on time scales. But as far as we know that, the stability observations of Ulam's type of non-linear Volterra integro-delay dynamic systems having noninstantaneous impulses are also not yet investigated .

Motivated by the work done in [30,49], the utmost purpose of this manuscript is to find different Hyers-Ulam and Hyers-Ulam-Rassias outcomes of stability for the following non-linear Volterra integrodynamic system of the form

$$
z^{\Delta}(\mathrm{t})=\mathrm{A}(\mathrm{t}) z(\mathrm{t})+\int_{\mathrm{t}_{0}}^{\mathrm{t}} \mathcal{K}(\mathrm{t}, \mathrm{s}, z(\mathrm{~s})) \Delta \mathrm{s}, z\left(\mathrm{t}_{0}\right)=z_{0},
$$

and for the following nonlinear Volterra integro-delay dynamic system with non-instantaneous impulses 
of the form

$$
\left\{\begin{array}{l}
\omega^{\Delta}(t)=M(t) \omega(t)+\int_{t_{0}}^{t} \mathcal{K}(t, s, \omega(s), \omega(h(s))) \Delta s, \quad t \in\left(s_{i}, t_{i+1}\right] \cap T_{S}, i=0,1, \ldots, m, \\
\omega(t)=g_{i}(t, \omega(t), \omega(h(t))), t \in\left(t_{i}, s_{i}\right] \cap T_{S}, i=1,2, \ldots, m, \\
\omega(t)=\alpha(t), \quad t \in\left[s_{0}-\lambda, s_{0}\right] \cap T_{S}, \\
\omega\left(t_{0}\right)=\alpha\left(t_{0}\right)=\omega_{0},
\end{array}\right.
$$

where $\lambda>0, A(t)$ and $M(t)$ are continuous and piecewise continuous on $T_{S}^{0}:=\left[t_{0}, t_{f}\right]_{T_{S}}$, respectively, $0=t_{0}=s_{0}<t_{1} \leqslant s_{1} \leqslant t_{2}<\cdots<t_{m} \leqslant s_{m} \leqslant t_{m} \leqslant t_{m+1}=t_{f}$ are pre-fixed numbers, $\mathcal{K}(t, s, z(s))$ and $\mathcal{K}(\mathrm{t}, \mathrm{s}, z(\mathrm{~s}), z(\mathrm{~h}(\mathrm{~s})))$ are continuous and piecewise continuous operators on $\Gamma=\left\{(\mathrm{t}, \mathrm{s}, z): \mathrm{t}_{0} \leqslant \mathrm{~s} \leqslant\right.$ $\left.t \leqslant t_{f}, z \in R^{\mathfrak{n}}\right\}$, respectively, $g_{i}:\left(t_{i}, s_{i}\right] \cap T_{S} \times R^{n} \times R^{n} \rightarrow R^{n}, i=1,2, \ldots, m$ are continuous functions, and $\phi:\left[s_{0}-\lambda, s_{0}\right] \cap T_{S} \rightarrow R^{n}$ is history function. Moreover, $h:\left[s_{0}-\lambda, t_{f}\right] \cap T_{S} \rightarrow\left(s_{i}, t_{i+1}\right] \cap T_{S}$ is a delay function with the consumption of continuity, additionally $h(t) \leqslant t$.

\section{Preliminaries}

In this section, we recall the main definitions and some basic notations of time scales calculus.

An arbitrary non-empty closed subset of real numbers $T_{S}$ is called a time scale. The forward jump operator $\Theta: T_{S} \rightarrow T_{S}$, backward jump operator $\rho: T_{S} \rightarrow T_{S}$, and graininess operator $\mu: T_{S} \rightarrow[0, \infty)$, are defined by:

$$
\Theta(s)=\inf \left\{t \in T_{S}: t>s\right\}, \quad \rho(s)=\sup \left\{t \in T_{S}: t<s\right\}, \quad \mu(s)=\Theta(s)-s,
$$

respectively. An arbitrary $t \in T_{S}$ is called left scattered (resp. left dense) when $t<\rho(t)$ (resp. $t=\rho(t)$ ). While, in case of $t<\Theta(t)$ (resp. $\Theta(t)=t$ ), we call $t$ is right scattered (resp. right dense). For a time scale $\mathrm{T}_{\mathrm{S}}$, the set of all limiting points $\mathrm{T}_{\mathrm{S}}{ }^{z}$ is called the derived set and illustrated as follows:

$$
T_{S}^{z}= \begin{cases}T_{S} \backslash\left(\rho\left(\sup T_{S}\right), \sup T_{S}\right], & \text { if } \sup T_{S}<\infty, \\ T_{S}, & \text { if } \sup T_{S}=\infty .\end{cases}
$$

The function $\mathcal{W}: T_{S} \rightarrow R$ is called regressive (resp. positively regressive) if $1+\mu(t) \mathcal{W}(t) \neq 0$, ( resp. $1+\mu(t) \mathcal{W}(t)>0) \quad \forall t \in T_{S}{ }^{z}$. The set of all right-dense continuous regressive functions (resp. rightdense continuous positively regressive functions) will be denoted by $\mathcal{R}_{\mathcal{G}}\left(T_{S}\right)\left(\operatorname{resp} . \mathcal{R}_{\mathcal{G}}\left(T_{S}\right)^{+}\right)$. The delta derivative of the function $W: T_{S} \rightarrow R$ on $t \in T_{S}^{z}$, is given by

$$
W^{\Delta}(t)=\lim _{s \rightarrow t, s \neq \Theta(t)} \frac{W(\Theta(t))-W(s)}{\Theta(t)-s} .
$$

For a rd-continuous function $W: T_{S} \rightarrow R$, the $\Delta$-integral is defined to be

$$
\int_{a}^{b} W(t) \Delta t=w(b)-w(a), \text { for all } a, b \in T_{S},
$$

where $w$ is the anti-derivative of $W$, i.e., $w^{\Delta}=W$ on $T_{S}^{z}$.

For $p \in \mathcal{R}_{\mathcal{G}}\left(T_{S}\right)$, the generalized exponential function is defined by

$$
e_{p}(a, b)=\exp \left(\int_{a}^{b} \alpha_{\mu(s)} p(s) \Delta s\right) \text { for all } a, b \in T_{S},
$$

while

$$
\alpha_{\mu(t)} p(t)= \begin{cases}\frac{\log (1+\mu(t) p(t))}{\mu(t)}, & \text { if } \mu(t) \neq 0, \\ p(t), & \text { if } \mu(t)=0,\end{cases}
$$

is the cylindrical transformation.

The fundamental matrix $\Psi_{M}\left(t, t_{0}\right)$ is the unique solution of the dynamic equation $\omega^{\Delta}(t)=M(t) \omega(t)$, $\omega\left(t_{0}\right)=\omega_{0}, t \in T_{S}{ }^{0}$. 


\section{Basic concepts and remarks}

Consider $C\left(T_{S}{ }^{0}, R^{n}\right)$ be the Banach space of continuous functions with norm $\|z\|=\sup _{t \in T_{S}{ }^{0}}\|z(t)\|$. Let $C\left(J, R^{n}\right)$ (resp. $P C\left(J, R^{n}\right)$ ) be the Banach space of all continuous functions (resp. the Banach space of piecewise continuous functions) with the norm $\|z\|_{\infty}=\sup _{J}\|z(t)\|, J=\left[s_{0}-\lambda, t_{f}\right] \cap T_{S}$ and R represents the set of real numbers. Finally, we denote by $P^{1}\left(J, R^{\mathfrak{n}}\right)=\left\{z \in P C\left(J, R^{\mathfrak{n}}\right): z^{\Delta} \in P C\left(J, R^{n}\right)\right\}$, the Banach space with norm $\|z\|_{1}=\sup \left\{\|z\|_{\infty},\left\|z^{\Delta}\right\|_{\infty}\right\}$. Here, we denote by $\|x\|=\sum_{i=1}^{n}\left|x_{i}\right|$ for $x=\left(x_{1}, \ldots, x_{n}\right) \in R^{n}$. Consider the following inequalities

$$
\begin{aligned}
& \left\|y^{\Delta}(t)-A(t) y(t)-\int_{t_{0}}^{t} \mathcal{K}(t, s, y(s)) \Delta s\right\| \leqslant \epsilon ; t \in T_{S}{ }^{0}, \\
& \left\|y^{\Delta}(t)-A(t) y(t)-\int_{t_{0}}^{t} \mathcal{K}(t, s, y(s)) \Delta s\right\| \leqslant \varphi(t) ; t \in T_{S}{ }^{0}, \\
& \left\{\begin{array}{l}
\left\|\phi^{\Delta}(t)-M(t) \phi(t)-\int_{t_{0}}^{t} \mathcal{K}(t, s, \phi(s), \phi(h(s))) \Delta s\right\| \leqslant \epsilon, t \in\left(s_{i}, t_{i+1}\right] \cap T_{S}, i=0,1, \ldots, m, \\
\left\|\phi(t)-g_{i}(t, \phi(t), \phi(h(t)))\right\| \leqslant \epsilon, t \in\left(t_{i}, s_{i}\right] \cap T_{S}, i=1,2, \ldots, m,
\end{array}\right. \\
& \left\{\begin{array}{l}
\left\|\phi^{\Delta}(t)-M(t) \phi(t)-\int_{t_{0}}^{t} \mathcal{K}(t, s, \phi(s), \phi(h(s))) \Delta s\right\| \leqslant \varphi(t), t \in\left(s_{i}, t_{i+1}\right] \cap T_{S}, i=0,1, \ldots, m, \\
\left\|\phi(t)-g_{i}(t, \phi(t), \phi(h(t)))\right\| \leqslant k, t \in\left(t_{i}, s_{i}\right] \cap T_{S}, i=1,2, \ldots, m,
\end{array}\right.
\end{aligned}
$$

where $\epsilon>0, \kappa \geqslant 0$ and $\varphi \in \mathrm{PC}\left(\mathrm{J}, \mathrm{R}^{+}\right)$is an increasing function.

Definition 3.1. Equation (1.1) is Hyers-Ulam stable on $T_{S}{ }^{0}$ if for every $y \in C\left(T_{S}{ }^{0}, R^{n}\right)$ satisfying (3.1), there exists a solution $y_{0} \in C\left(T_{S}^{0}, R^{n}\right)$ of (1.1) with $\left\|y_{0}(t)-y(t)\right\| \leqslant K \epsilon, K>0, \forall t \in T_{S}^{0}$.

Definition 3.2. Equation (1.1) is Hyers-Ulam-Rassias stable on $T_{S}^{0}$ if for every $y \in C\left(T_{S}{ }^{0}, R^{n}\right)$ satisfying (3.2), there exists a solution $y_{0} \in C\left(T_{S}{ }^{0}, R^{n}\right)$ of (1.1) with $\left\|y_{0}(t)-y(t)\right\| \leqslant K \varphi(t), K>0, \forall t \in T_{S}{ }^{0}$.

Definition 3.3. Equation (1.2) is said to be stable in the sense of Hyers-Ulam, if for every $\epsilon>0$ and $\phi \in$ $P C^{1}\left(J, R^{n}\right)$ satisfying (3.3), there exists a solution $\phi_{0} \in P C^{1}\left(J, R^{n}\right)$ of (1.2) such that $\left\|\phi_{0}(t)-\phi(t)\right\| \leqslant K \epsilon$ for all $t \in J$. Here $K$ is a positive number that depends on $\epsilon$.

Definition 3.4. Equation (1.2) is said to be stable in the sense of Hyers-Ulam-Rassias, provided for every $(\varphi, \kappa) \in P C\left(J, R^{+}\right) \times R^{\geqslant 0}$ and for each $\phi \in P C^{1}\left(J, R^{n}\right)$ satisfying (3.4), there exists a solution $\phi_{0} \in \mathrm{PC}^{1}\left(J, R^{n}\right)$ of (1.2) such that the inequality $\left\|\phi_{0}(t)-\phi(t)\right\| \leqslant M \varphi(t)$ is true for all $t \in J$. Here $M>0$ depends on $(\varphi, \kappa)$.

Definition 3.5. In a metric space $(X ; d)$, a mapping $\Lambda: X \rightarrow X$ is said to be Picard operator if it has precisely a unique fixed point $x^{*} \in X$, so that for every $x \in X$, the sequence $\left\{\Lambda^{(n)}(x)\right\}_{n \in N}$ converges to $x^{*}$.

Lemma 3.6 ([8, Grönwall's inequality, Corollary 6.7]). Let $y$ be the $r d$-continuous function, $p \in \mathcal{R}_{\mathcal{G}}\left(\mathcal{T}_{\mathcal{S}}\right)^{+}, p \geqslant$ 0 and $\alpha \in \mathbb{R}$. Then

$$
y(t) \leqslant \alpha+\int_{t_{0}}^{t} y(u) p(u) \Delta u, \forall t \in \mathcal{T}_{\mathcal{S}}
$$

implies

$$
y(t) \leqslant \alpha e_{p}\left(t, t_{0}\right), \forall t \in \mathcal{T}_{\mathcal{S}} .
$$

Lemma 3.7 ([20]). Suppose $\tau \in \mathrm{T}_{\mathrm{S}}^{+}, \mathrm{y}, \mathrm{b} \in \mathcal{R}_{\mathcal{G}}\left(\mathrm{T}_{\mathrm{S}}{ }^{+}\right), \mathrm{p} \in \mathcal{R}_{\mathcal{G}}\left(\mathrm{T}_{\mathrm{S}}{ }^{+}\right)^{+}$and $\mathrm{c}, \mathrm{b}_{\mathrm{k}} \in \mathrm{R}^{+}, \mathrm{k}=1,2, \ldots$, so

$$
y(t) \leqslant c+\int_{\tau}^{t} p(s) y(s) \Delta s+\sum_{\tau<t_{k}<t} b_{k} y\left(t_{k}\right),
$$

implies

$$
y(t) \leqslant c \prod_{\tau<t_{k}<t}\left(1+b_{k}\right) e_{p}(t, \tau), t \geqslant \tau
$$


Lemma 3.8 ([26, Abstract Grönwall lemma]). Let $(X, \mathrm{~d}, \leqslant)$ be an ordered metric space and let $x^{*}$ be a fixed point for the increasing mapping $\Lambda: X \rightarrow X$. So, being arbitrary $x \in X, x \leqslant \Lambda(x)$ entails $x \leqslant x^{*}$ and $x \geqslant \Lambda(x)$ entails $x \geqslant x^{*}$, where $x^{*}$ denotes the fixed point in $\Lambda$.

Remark 3.9. A function $y \in C\left(T_{S}{ }^{0}, R^{n}\right)$ satisfies (3.1) if and only if there is a function $h \in C\left(T_{S}{ }^{0}, R^{n}\right)$ such that $\|h(t)\| \leqslant \epsilon$ for all $t \in T_{S}{ }^{0}$ and

$$
y^{\Delta}(t)=A(t) y(t)+\int_{t_{0}}^{t} \mathcal{K}(t, s, y(s)) \Delta s+h(t), y\left(t_{0}\right)=y_{0}
$$

We do similar remark for (3.2).

Lemma 3.10. Every $y \in C\left(T_{S}{ }^{0}, R^{n}\right)$ that satisfies (3.1) also comes out perfect on the following inequality

$$
\left\|y(t)-\Psi_{A}\left(t, t_{0}\right) y_{0}-\int_{t_{0}}^{t} \Psi_{A}(t, \Theta(s)) \int_{t_{0}}^{s} \mathcal{K}(s, u, y) \Delta u \Delta s\right\| \leqslant C\left(t_{f}-t_{0}\right) \epsilon,
$$

for $\mathrm{t} \in \mathrm{T}_{\mathrm{S}}{ }^{0}$. Here $\mathrm{C}$ is the bound of fundamental matrix $\Psi_{\mathrm{A}}(\mathrm{t}, \Theta(\mathrm{s}))$.

Proof. If $\mathrm{y} \in \mathrm{C}\left(\mathrm{T}_{\mathrm{S}}{ }^{0}, \mathrm{R}^{\mathrm{n}}\right)$ satisfies (3.1), then by Remark 3.9, we have

$$
y^{\Delta}(t)=A(t) y(t)+\int_{t_{0}}^{t} \mathcal{K}(t, s, y(s)) \Delta s+h(t), y\left(t_{0}\right)=y_{0}
$$

Then

$$
y(t)=\Psi_{A}\left(t, t_{0}\right) y_{0}+\int_{t_{0}}^{t} \Psi_{A}(t, \Theta(s)) \int_{t_{0}}^{s} \mathcal{K}(s, u, y) \Delta u \Delta s+\int_{t_{0}}^{t} \Psi_{A}(t, \Theta(s)) h(s) \Delta s
$$

So,

$$
\begin{aligned}
\left\|y(t)-\Psi_{A}\left(t, t_{0}\right) y_{0}-\int_{t_{0}}^{t} \Psi_{A}(t, \Theta(s)) \int_{t_{0}}^{s} \mathcal{K}(s, u, y) \Delta u \Delta s\right\| & \leqslant \int_{t_{0}}^{t}\left\|\Psi_{A}(t, \Theta(s))\right\|\|h(s)\| \Delta s \\
& \leqslant C\left(t-t_{0}\right) \epsilon \leqslant C\left(t_{f}-t_{0}\right) \epsilon .
\end{aligned}
$$

We have similar remarks for (3.2).

Remark 3.11. A function $\phi \in \mathrm{PC}^{1}\left(\mathrm{~J}, \mathrm{R}^{\mathrm{n}}\right)$ satisfies inequality (3.3) (resp. inequality (3.4)) if and only if there exist a function $f \in P C^{1}\left(J, R^{n}\right)$ and a finite sequence $\left\{f_{k}: k=1, \ldots, m\right\} \subset R^{n}$ (dependent on $\phi$ ) such that $\|f(t)\| \leqslant \epsilon$ for all $t \in J$ and $\left\|f_{i}\right\| \leqslant \epsilon$ (resp. $\left\|f_{i}\right\| \leqslant k$ ) for every $i=1,2, \ldots, m$ and

$$
\left\{\begin{array}{l}
\phi^{\Delta}(t)=M(t) \phi(t)+\int_{t_{0}}^{t} \mathcal{K}(t, s, \phi(s), \phi(h(s))) \Delta s+f(t), t \in\left(s_{i}, t_{i+1}\right] \cap T_{S}, i=0,1, \ldots, m, \\
\phi(t)=g_{i}(t, \phi(t), \phi(h(t)))+f_{i}, t \in\left(t_{i}, s_{i}\right] \cap T_{S}, i=1,2, \ldots, m .
\end{array}\right.
$$

Lemma 3.12. If $\phi \in \mathrm{PC}^{1}\left(\mathrm{~J}, \mathrm{R}^{\mathrm{n}}\right)$ satisfies inequality (3.3) (resp. inequality (3.4)), then the following inequalities

$$
\left\{\begin{array}{l}
\left\|\phi(t)-\phi_{0}-\Psi_{M}\left(t, t_{0}\right) \phi_{0}-\int_{s_{i}}^{t} \Psi_{M}(t, \Theta(s)) \int_{s_{0}}^{s} \mathcal{K}(s, u, \phi(u), \phi(h(u))) \Delta u \Delta s-g_{i}(t, \phi(t), \phi(h(t)))\right\| \\
\quad \leqslant\left(C t_{f}-C s_{i}+m\right) \epsilon, t \in\left(s_{i}, t_{i+1}\right] \cap T_{S}, i=1,2, \ldots, m, \\
\left\|\phi(t)-g_{i}(t, \phi(t), \phi(h(t)))\right\| \leqslant m \epsilon,(\text { resp. } m k), t \in\left(t_{i}, s_{i}\right] \cap T_{S}, i=1,2, \ldots, m,
\end{array}\right.
$$

are true. Here $\mathrm{C}$ is the bound of fundamental matrix $\Psi_{M}(t, \Theta(s))$. 
Proof. If $\phi \in \mathrm{PC}^{1}\left(\mathrm{~J}, \mathrm{R}^{\mathfrak{n}}\right)$ satisfies (3.3), then by Remark 3.11, we have

$$
\left\{\begin{array}{l}
\phi^{\Delta}(t)=M(t) \phi(t)+\int_{t_{0}}^{t} \mathcal{K}(t, s, \phi(s), \phi(h(s))) \Delta s+f(t), t \in\left(s_{i}, t_{i+1}\right] \cap T_{S}, i=0,1, \ldots, m, \\
\phi(t)=g_{i}(t, \phi(t), \phi(h(t)))+f_{i}, t \in\left(t_{i}, s_{i}\right] \cap T_{S}, i=1,2, \ldots, m .
\end{array}\right.
$$

Clearly the solution of (3.5) is given as

$$
\phi(t)=\left\{\begin{array}{c}
\phi_{0}+\Psi_{M}\left(t, t_{0}\right) \phi_{0}+\int_{s_{i}}^{t} \Psi_{M}(t, \Theta(s))\left(\int_{s_{0}}^{s} \mathcal{K}(s, u, \phi(u), \phi(h(u))) \Delta u+f(s)\right) \Delta s+g_{i}(t, \phi(t), \phi(h(t))), \\
t \in\left(s_{i}, t_{i+1}\right] \cap T_{S}, i=1,2, \ldots, m, \\
g_{i}(t, \phi(t), \phi(h(t)))+f_{i}, t \in\left(t_{i}, s_{i}\right] \cap T_{S}, i=1,2, \ldots, m .
\end{array}\right.
$$

For $t \in\left(s_{i}, t_{i+1}\right] \cap T_{S}, i=1,2, \ldots, m$, we get

$$
\begin{gathered}
\left\|\phi(t)-\phi_{0}-\Psi_{M}\left(t, t_{0}\right) \phi_{0}-\int_{s_{i}}^{t} \Psi_{M}(t, \Theta(s)) \int_{s_{0}}^{s} \mathcal{K}(s, u, \phi(u), \phi(h(u))) \Delta u \Delta s-g_{i}(t, \phi(t), \phi(h(t)))\right\| \\
\leqslant \int_{s_{i}}^{t}\left\|\Psi_{M}(t, \Theta(s))\right\|\|f(s)\| d s+\sum_{i=1}^{m}\left\|f_{i}\right\| \leqslant\left(C t-C s_{i}+m\right) \epsilon \leqslant\left(C t_{f}-C s_{i}+m\right) \epsilon .
\end{gathered}
$$

Proceeding as above we derive

$$
\left\|\phi(t)-g_{i}(t, \phi(t), \phi(h(t)))\right\| \leqslant m \epsilon, t \in\left(t_{i}, s_{i}\right] \cap T_{S}, i=1,2, \ldots, m .
$$

We have similar processions for (3.4).

\section{Hyers-Ulam stability of equation (1.1)}

Now we are going to give our result on Hyers-Ulam stability. First we assume some of the following conditions:

$\left(C_{1}\right)$ the function $\mathcal{K}$ is continuous with the Lipschitz condition $\left\|\mathcal{K}\left(t, s, x_{1}\right)-\mathcal{K}\left(t, s, x_{2}\right)\right\| \leqslant L_{k}\left\|x_{1}-x_{2}\right\|$, $\mathrm{L}_{\mathrm{k}}>0$, for $\mathrm{t}_{0} \leqslant s \leqslant \mathrm{t} \leqslant \mathrm{t}_{\mathrm{f}}$ and for all $\mathrm{x}_{1}, \mathrm{x}_{2} \in \mathrm{R}^{\mathrm{n}}$

$\left(C_{2}\right) \sup _{t \in T_{s}} \int_{t_{0}}^{t}\left\|\Psi_{A}(t, \Theta(s))\right\| \int_{t_{0}}^{s} L_{k} \Delta u \Delta s<1$;

$\left(\mathrm{C}_{3}\right) \varphi \in \mathrm{C}\left(\mathrm{T}_{\mathrm{S}}^{0}, \mathrm{R}^{\mathfrak{n}}\right)$ is an increasing function such that for some $\rho>0$

$$
\int_{t_{0}}^{t} \varphi(s) \Delta s \leqslant \rho \varphi(t) .
$$

Theorem 4.1. If conditions $\left(C_{1}\right)-\left(C_{2}\right)$ hold, then equation (1.1) has precisely a unique solution in $C\left(T_{S}{ }^{0}, R^{n}\right)$.

Proof.

i) Define an operator $\Lambda: C\left(T_{S}^{0}, R^{n}\right) \rightarrow C\left(T_{S}^{0}, R^{n}\right)$ by

$$
(\Lambda z)(\mathrm{t})=\Psi_{\mathrm{A}}\left(\mathrm{t}, \mathrm{t}_{0}\right) z_{0}+\int_{\mathrm{t}_{0}}^{\mathrm{t}} \Psi_{\mathrm{A}}(\mathrm{t}, \Theta(\mathrm{s})) \int_{\mathrm{t}_{0}}^{\mathrm{s}} \mathcal{K}(\mathrm{s}, \mathrm{u}, z) \Delta \mathrm{u} \Delta \mathrm{s} .
$$

Now for any $z_{1}, z_{2} \in \mathrm{C}\left(\mathrm{T}_{\mathrm{S}}{ }^{0}, \mathrm{R}^{\mathfrak{n}}\right)$, we have

$$
\left\|\left(\Lambda z_{1}\right)(\mathrm{t})-\left(\Lambda z_{2}\right)(\mathrm{t})\right\|=\left\|\int_{\mathrm{t}_{0}}^{\mathrm{t}} \Psi_{\mathrm{A}}(\mathrm{t}, \Theta(\mathrm{s})) \int_{\mathrm{t}_{0}}^{\mathrm{s}}\left(\mathcal{K}\left(\mathrm{s}, \mathrm{u}, z_{1}\right)-\mathcal{K}\left(\mathrm{s}, \mathrm{u}, z_{2}\right)\right) \Delta \mathrm{u} \Delta \mathrm{s}\right\|
$$




$$
\begin{aligned}
& \leqslant \sup _{t \in T_{s}{ }^{0}} \int_{t_{0}}^{t}\left\|\Psi_{A}(t, \Theta(s))\right\| \int_{t_{0}}^{s}\left\|\left(\mathcal{K}\left(s, u, z_{1}\right)-\mathcal{K}\left(s, u, z_{2}\right)\right)\right\| \Delta u \Delta s \\
& \leqslant \sup _{t \in T_{S} 0} \int_{t_{0}}^{t}\left\|\Psi_{A}(t, \Theta(s))\right\| \int_{t_{0}}^{s} L_{k}\left\|z_{1}(u)-z_{2}(u)\right\| \Delta u \Delta s \\
& \leqslant \sup _{t \in T_{S}{ }^{0}}\left\|z_{1}(t)-z_{2}(t)\right\| \sup _{t \in T_{S} 0} \int_{t_{0}}^{t}\left\|\Psi_{A}(t, \Theta(s))\right\| \int_{t_{0}}^{s} L_{k} \Delta u \Delta s \\
& \leqslant\left\|z_{1}-z_{2}\right\| \sup _{t \in T_{S}} \int_{t_{0}}^{t}\left\|\Psi_{A}(t, \Theta(s))\right\| \int_{t_{0}}^{s} L_{k} \Delta u \Delta s .
\end{aligned}
$$

Following from $\left(C_{2}\right)$, the operator is strictly contractive and hence a Picard operator on $C\left(T_{S}{ }^{0}, R^{n}\right)$. From (3.1), it follows that the unique fixed point of this operator is in fact the unique solution of (1.1) in $\mathrm{C}\left(\mathrm{T}_{\mathrm{S}}{ }^{0}, \mathrm{R}^{\mathrm{n}}\right)$.

Theorem 4.2. If conditions $\left(C_{1}\right)-\left(C_{2}\right)$ hold, then equation (1.1) has Hyers-Ulam stability on $\mathrm{T}_{\mathrm{S}}{ }^{0}$.

Proof. Let $\mathrm{y} \in \mathrm{C}\left(\mathrm{T}_{\mathrm{S}}{ }^{0}, \mathrm{R}^{\mathrm{n}}\right)$ be a solution to (3.1). The unique solution $z \in \mathrm{C}\left(\mathrm{T}_{\mathrm{S}}{ }^{0}, \mathrm{R}^{\mathfrak{n}}\right)$ of the equation (1.1) is given by

$$
z(t)=\Psi_{A}\left(t, t_{0}\right) z_{0}+\int_{t_{0}}^{t} \Psi_{A}(t, \Theta(s)) \int_{t_{0}}^{s} \mathcal{K}(s, u, z) \Delta u \Delta s
$$

Now by using Lemma 3.9,

$$
\begin{aligned}
\|y(t)-z(t)\| \leqslant & \left\|y(t)-\Psi_{A}\left(t, t_{0}\right) y_{0}-\int_{t_{0}}^{t} \Psi_{A}(t, \Theta(s)) \int_{t_{0}}^{s} \mathcal{K}(s, u, y) \Delta u \Delta s\right\| \\
& +\int_{t_{0}}^{t}\left\|\Psi_{A}(t, \Theta(s))\right\| \int_{t_{0}}^{s}\|(\mathcal{K}(s, u, y)-K(s, u, z))\| \Delta u \Delta s \\
\leqslant & C\left(t_{f}-t_{0}\right) \epsilon+\int_{t_{0}}^{t}\left\|\Psi_{A}(t, \Theta(s))\right\| \int_{t_{0}}^{s} L_{k}\|y(u)-z(u)\| \Delta u \Delta s .
\end{aligned}
$$

Next, we show that the operator $\mathrm{T}: \mathrm{C}\left(\mathrm{T}_{\mathrm{S}}{ }^{0}, \mathrm{R}^{+}\right) \rightarrow \mathrm{C}\left(\mathrm{T}_{\mathrm{S}}{ }^{0}, \mathrm{R}^{+}\right)$given below is an increasing Picard operator,

$$
(T g)(t)=C\left(t_{f}-t_{0}\right) \epsilon+\int_{t_{0}}^{t}\left\|\Psi_{A}(t, \Theta(s))\right\| \int_{t_{0}}^{s} L_{k} g(u) \Delta u \Delta s
$$

For any $g_{1}, g_{2} \in C\left(T_{S}{ }^{0}, R^{+}\right)$, we have

$$
\begin{aligned}
\left\|\left(T g_{1}\right)(t)-\left(T g_{2}\right)(t)\right\| & =\left\|\int_{t_{0}}^{t}\right\| \Psi_{A}(t, \Theta(s))\left\|\int_{t_{0}}^{s} L_{k}\left(g_{1}(u)-g_{2}(u)\right) \Delta u \Delta s\right\| \\
& \leqslant \sup _{t \in T_{s}} \int_{t_{0}}^{t}\left\|\Psi_{A}(t, \Theta(s))\right\| \int_{t_{0}}^{s} L_{k}\left\|g_{1}(u)-g_{2}(u)\right\| \Delta u \Delta s \\
& \leqslant \sup _{t \in T_{s}{ }^{0}}\left\|g_{1}(t)-g_{2}(t)\right\| \sup _{t \in T_{s}} \int_{t_{0}}^{t}\left\|\Psi_{A}(t, \Theta(s))\right\| \int_{t_{0}}^{s} L_{k} \Delta u \Delta s \\
& \leqslant\left\|g_{1}-g_{2}\right\| \sup _{t \in T_{s} 0} \int_{t_{0}}^{t}\left\|\Psi_{A}(t, \Theta(s))\right\| \int_{t_{0}}^{s} L_{k} \Delta u \Delta s .
\end{aligned}
$$

Since $\left(\sup _{t \in T_{S}} \int_{t_{0}}^{t}\left\|\Psi_{A}(t, \Theta(s))\right\| \int_{t_{0}}^{s} L_{k} \Delta u \Delta s\right)<1$, so the operator is contractive on $C\left(T_{S}{ }^{0}, R^{+}\right)$. Applying Banach contraction principle, $T$ is Picard operator with unique fixed point $g^{*} \in C\left(T_{S}{ }^{0}, R^{+}\right)$, i.e.,

$$
g^{*}(t)=C\left(t_{f}-t_{0}\right) \epsilon+\int_{t_{0}}^{t}\left\|\Psi_{A}(t, \Theta(s))\right\| \int_{t_{0}}^{s} L_{k} g^{*}(u) \Delta u \Delta s
$$


For some $M_{k}>0$, we have $\left\|\Psi_{A}(t, \Theta(s))\right\|=\sup _{t \in T_{S} 0}\left\|\Psi_{A}(t, \Theta(s))\right\| \leqslant M_{k}$, so

$$
g^{*}(t) \leqslant C\left(t_{f}-t_{0}\right) \epsilon+\int_{t_{0}}^{t} M_{k} \int_{t_{0}}^{s} L_{k} g^{*}(u) \Delta u \Delta s .
$$

By Lemma 3.6, we get

$$
g^{*}(t) \leqslant C\left(t_{f}-t_{0}\right) \in e_{P}\left(t, t_{0}\right),
$$

where $P(s)=\int_{t_{0}}^{s} L_{k} M_{k} \Delta u$. If we set $g(t)=\|y(t)-z(t)\|$, then from (4.1), $g(t) \leqslant(T g)(t)$ from which by using abstract Grönwall lemma, it follows that $g(t) \leqslant g^{*}(t)$, thus

$$
\|y(t)-z(t)\| \leqslant C\left(t_{f}-t_{0}\right) \epsilon e_{P}\left(t, t_{0}\right)
$$

Similarly, by following the same process, we can prove that:

Theorem 4.3. If conditions $\left(\mathrm{C}_{1}\right)-\left(\mathrm{C}_{3}\right)$ hold, then equation (1.1) has Hyers-Ulam-Rassias stability on $\mathrm{T}_{\mathrm{S}}{ }^{0}$.

\section{Hyers-Ulam stability of equation (1.2)}

Onward we will state our major results. The first solution to be establish is Hyers-Ulam stability. First we assume some of the following conditions:

$\left(A_{1}\right)$ the function $\mathcal{K}$ is piecewise continuous with the Lipschitz condition $\left\|\mathcal{K}\left(t, s, x_{1}, x_{2}\right)-\mathcal{K}\left(t, s, y_{1}, y_{2}\right)\right\| \leqslant$ $\sum_{k=1}^{2} L\left\|x_{k}-y_{k}\right\|, L>0$, for all $t \in\left(s_{i}, t_{i+1}\right] \cap T_{S}, i=0,1, \ldots, m$ and $x_{k}, y_{k} \in R^{n}, k \in\{1,2\} ;$

$\left(A_{2}\right) g_{i}:\left(t_{i}, s_{i}\right] \cap T_{S} \times R^{n} \times R^{n} \rightarrow R^{n}$ satisfies the Lipschitz condition $\left\|g_{i}\left(t, u_{1}, u_{2}\right)-g_{i}\left(t, v_{1}, v_{2}\right)\right\| \leqslant$ $\sum_{k=1}^{2} L_{g_{i}}\left\|u_{k}-v_{k}\right\|, L_{g_{i}}>0$, for all $t \in\left(t_{i}, s_{i}\right] \cap T_{S}, i=1,2, \ldots, m$ and $u_{1}, u_{2}, v_{1}, v_{2} \in R$;

$\left(\mathrm{A}_{3}\right)\left(\sum_{0<s_{i}<\mathrm{t}} 2 \mathrm{~L}_{\mathrm{i}}+2 \mathrm{CL} \int_{s_{\mathrm{i}}}^{\mathrm{t}} \int_{s_{0}}^{s} \Delta \mathrm{u} \Delta \mathrm{s}\right)<1, i=1,2, \ldots, \mathrm{m}$;

$\left(\mathrm{A}_{4}\right) \varphi \in \mathrm{PC}\left(\mathrm{J}, \mathrm{R}^{+}\right)$is increasing so that for some $\rho>0$

$$
\int_{t_{0}}^{t} \varphi(r) \Delta r \leqslant \rho \varphi(t)
$$

Theorem 5.1. If conditions $\left(\mathrm{A}_{1}\right)-\left(\mathrm{A}_{3}\right)$ hold, then equation (1.2) has precisely a unique solution in $\mathrm{PC}^{1}\left(\mathrm{~J}, \mathbb{R}^{\mathrm{n}}\right)$.

Proof.

i) Determine an operator $\Lambda: P C\left(J, R^{n}\right) \rightarrow P C\left(J, R^{n}\right)$, as

$$
(\Lambda \omega)(t)=\left\{\begin{array}{l}
\alpha(t), t \in\left[s_{0}-\lambda, s_{0}\right] \cap T_{S}, \\
g_{i}\left(s_{i}, \omega\left(s_{i}\right), \omega\left(h\left(s_{i}\right)\right)\right), t \in\left(t_{i}, s_{i}\right] \cap T_{S}, i=1,2, \ldots, m, \alpha \in(0,1), \\
\alpha\left(t_{0}\right)+\Psi_{M}\left(t, t_{0}\right) \omega_{0}+g_{i}\left(s_{i}, \omega\left(s_{i}\right), \omega\left(h\left(s_{i}\right)\right)\right) \\
\quad+\int_{s_{i}}^{t} \Psi_{M}(t, \Theta(s)) \int_{s_{0}}^{s} \mathcal{K}(s, u, \omega(u), \omega(h(u))) \Delta u \Delta s, \\
\quad t \in\left(s_{i}, t_{i+1}\right] \cap T_{S}, i=1,2, \ldots, m, \alpha \in(0,1) .
\end{array}\right.
$$

For any $\omega_{1}, \omega_{2} \in P C\left(J, R^{n}\right), t \in\left(s_{i}, t_{i+1}\right] \cap T_{S}, i=1,2, \ldots, m$, we have

$$
\begin{aligned}
\left\|\left(\Lambda \omega_{1}\right)(t)-\left(\Lambda \omega_{2}\right)(t)\right\| \leqslant & \left\|g_{i}\left(s_{i}, \omega_{1}\left(s_{i}\right), \omega_{1}\left(h\left(s_{i}\right)\right)\right)-g_{i}\left(s_{i}, w_{2}\left(s_{i}\right), \omega_{2}\left(h\left(s_{i}\right)\right)\right)\right\| \\
& +\int_{s_{i}}^{t}\left\|\Psi_{M}(t, \Theta(s))\right\| \| \int_{s_{0}}^{s} \mathcal{K}\left(s, u, \omega_{1}(u), \omega_{1}(h(u))\right) \Delta u \Delta s
\end{aligned}
$$




$$
\begin{aligned}
& -\int_{s_{0}}^{s} \mathcal{K}\left(s, u, \omega_{2}(u), \omega_{2}(h(u))\right) \Delta u \Delta s \| \\
\leqslant & L_{i}\left\|\omega_{1}\left(s_{i}\right)-\omega_{2}\left(s_{i}\right)\right\|+L_{i}\left\|\omega_{1}\left(h\left(s_{i}\right)\right)-\omega_{2}\left(h\left(s_{i}\right)\right)\right\| \\
& +L \int_{s_{i}}^{t}\left\|\Psi_{M}(t, \Theta(s))\right\| \int_{s_{0}}^{s}\left\|\omega_{1}(u)-\omega_{2}(u)\right\| \Delta u \Delta s \\
& +\mathrm{L} \int_{s_{i}}^{t}\left\|\Psi_{M}(t, \Theta(s))\right\| \int_{s_{0}}^{s}\left\|\omega_{1}(h(u))-\omega_{2}(h(u))\right\| \Delta u \Delta s \\
\leqslant & \sum_{0<s_{i}<t} L_{i}\left\|\omega_{1}\left(s_{i}\right)-\omega_{2}\left(s_{i}\right)\right\|+\sum_{0<s_{i}<t} L_{i}\left\|\omega_{1}\left(h\left(s_{i}\right)\right)-\omega_{2}\left(h\left(s_{i}\right)\right)\right\| \\
& +2 C L \int_{s_{i}}^{t} \int_{s_{0}}^{s}\left\|\omega_{1}-\omega_{2}\right\|_{\infty} \Delta u \Delta s \\
\leqslant & \sum_{0<s_{i}<t} 2 L_{i}\left\|\omega_{1}-\omega_{2}\right\|_{\infty}+2 C L \int_{s_{i}}^{t} \int_{s_{0}}^{s}\left\|\omega_{1}-\omega_{2}\right\|_{\infty} \Delta u \Delta s \\
\leqslant & \left(\sum_{0<s_{i}<t} 2 L_{i}+2 C L \int_{s_{i}}^{t} \int_{s_{0}}^{s} \Delta u \Delta s\right)\left\|\omega_{1}-\omega_{2}\right\|_{\infty} .
\end{aligned}
$$

According to $\left(A_{3}\right)$, we are dealing here with the strictly contractive operator on $\left(s_{i}, t_{i+1}\right] \cap T_{S}, i=$ $1,2, \ldots, m$, and hence a Picard operator on $P C\left(J, R^{n}\right)$. Regarding to (5.1), it shows that the unique solution of equation (1.2) in $\operatorname{PC}^{1}\left(J, R^{n}\right)$ is in fact the unique fixed point of this operator.

Theorem 5.2. If conditions $\left(\mathrm{A}_{1}\right)-\left(\mathrm{A}_{3}\right)$ hold then equation (1.2) has Hyers-Ulam stability on $\mathrm{J}$.

Proof. Assume that (3.1) has a solution $\operatorname{PC}^{1}\left(J, R^{n}\right)$. Then for dynamic equation (1.2), we have the unique solution

$$
\omega(t)=\left\{\begin{array}{l}
\alpha(t), t \in\left[s_{0}-\lambda, s_{0}\right] \cap T_{S}, \\
g_{i}(t, \omega(t), \omega(h(t))), t \in\left(t_{i}, s_{i}\right] \cap T_{S}, i=1,2, \ldots, m, \alpha \in(0,1), \\
\alpha\left(t_{0}\right)+\Psi_{M}\left(t, t_{0}\right) \omega_{0}+g_{i}\left(s_{i}, \omega\left(s_{i}\right), \omega\left(h\left(s_{i}\right)\right)\right)+\int_{s_{i}}^{t} \Psi_{M}(t, \Theta(s)) \int_{s_{0}}^{s} \mathcal{K}(s, u, \omega(u), \omega(h(u))) \Delta u \Delta s, \\
\quad t \in\left(s_{i}, t_{i+1}\right] \cap T_{S}, i=1,2, \ldots, m, \alpha \in(0,1) .
\end{array}\right.
$$

We observe that for all $t \in\left(s_{i}, t_{i+1}\right] \cap T_{S}, i=1,2, \ldots, m$, using Lemma 3.12, we have

$$
\begin{aligned}
\|\phi(t)-\omega(t)\| \leqslant & \| \phi(t)-\phi_{0}-\Psi_{M}\left(t, t_{0}\right) \phi_{0}-\int_{s_{i}}^{t} \Psi_{M}(t, \Theta(s)) \int_{s_{0}}^{s} \mathcal{K}(s, u, \phi(u), \phi(h(u))) \Delta u \Delta s \\
& -g_{i}(t, \phi(t), \phi(h(t)))\|+\| g_{i}\left(s_{i}, \phi\left(s_{i}\right), \phi\left(h\left(s_{i}\right)\right)\right)-g_{i}\left(s_{i}, \omega\left(s_{i}\right), \omega\left(h\left(s_{i}\right)\right)\right) \| \\
& +\int_{s_{i}}^{t}\left\|\Psi_{M}(t, \Theta(s))\right\| \int_{s_{0}}^{s}\|\mathcal{K}(s, u, \phi(u), \phi(h(u)))-\mathcal{K}(s, u, \omega(u), \omega(h(u)))\| \Delta u \Delta s \\
\leqslant & \left(m+C t_{f}-C s_{i}\right) \epsilon+\sum_{0<s_{i}<t} L_{i}\left\|\phi\left(s_{i}\right)-\omega\left(s_{i}\right)\right\|+\sum_{0<s_{i}<t} L_{i}\left\|\phi\left(h\left(s_{i}\right)\right)-\omega\left(h\left(s_{i}\right)\right)\right\| \\
& +C L \int_{s_{i}}^{t} \int_{s_{0}}^{s}\|\phi(u)-\omega(u)\| \Delta u \Delta s+C L \int_{s_{i}}^{t} \int_{s_{0}}^{s}\|\phi(h(u))-\omega(h(u))\| \Delta u \Delta s .
\end{aligned}
$$

Next, we show that the operator $\mathrm{T}: \mathrm{PC}\left(\mathrm{J}, \mathrm{R}^{+}\right) \rightarrow \mathrm{PC}\left(\mathrm{J}, \mathrm{R}^{+}\right)$given below is an increasing Picard operator:

$$
(T g)(t)=\left(m+C t_{f}-C s_{i}\right) \epsilon+\sum_{0<s_{i}<t} L_{i} g\left(s_{i}\right)+\sum_{0<s_{i}<t} L_{i} g\left(h\left(s_{i}\right)\right)
$$




$$
+\mathrm{CL} \int_{s_{i}}^{t} \int_{s_{0}}^{s} g(u) \Delta u \Delta s+C L \int_{s_{i}}^{t} \int_{s_{0}}^{s} g(h(u)) \Delta u \Delta s .
$$

For any $g_{1}, g_{2} \in P C\left(J, R^{+}\right), t \in\left(s_{i}, t_{i+1}\right] \cap T_{S}, i=1,2, \ldots, m$, we have

$$
\begin{aligned}
\left\|\left(T g_{1}\right)(t)-\left(T g_{2}\right)(t)\right\| \leqslant & \sum_{0<s_{i}<t} L_{i}\left\|g_{1}\left(s_{i}\right)-g_{2}\left(s_{i}\right)\right\|+\sum_{0<s_{i}<t} L_{i}\left\|g_{1}\left(h\left(s_{i}\right)\right)-g_{2}\left(h\left(s_{i}\right)\right)\right\| \\
& +C L \int_{s_{i}}^{t} \int_{s_{0}}^{s}\left\|g_{1}(u)-g_{2}(u)\right\| \Delta u \Delta s+C L \int_{s_{i}}^{t} \int_{s_{0}}^{s}\left\|g_{1}(h(u))-g_{2}(h(u))\right\| \Delta u \Delta s \\
\leqslant & \sum_{0<s_{i}<t} 2 L_{i}\left\|g_{1}-g_{2}\right\|_{\infty}+2 C L \int_{s_{i}}^{t} \int_{s_{0}}^{s}\left\|g_{1}-g_{2}\right\|_{\infty} \Delta u \Delta s \\
\leqslant & \left(\sum_{0<s_{i}<t} 2 L_{i}+2 C L \int_{s_{i}}^{t} \int_{s_{0}}^{s} \Delta u \Delta s\right)\left\|g_{1}-g_{2}\right\|_{\infty} .
\end{aligned}
$$

Again according to $\left(A_{3}\right)$, we are dealing here with the strictly contractive operator on $\left(s_{i}, t_{i+1}\right] \cap T_{S}, i=$ $1,2, \ldots, \mathrm{m}$ and hence a Picard operator on $\mathrm{PC}\left(\mathrm{J}, \mathrm{R}^{+}\right)$. Banach fixed point theorem imply, $\mathrm{T}$ is Picard operator having unique fixed point $g^{*} \in P C\left(J, R^{+}\right)$, i.e.,

$$
\begin{aligned}
g^{*}(t)= & \left(m+C t_{f}-C s_{i}\right) \epsilon+\sum_{0<s_{i}<t} L_{i} g^{*}\left(s_{i}\right)+\sum_{0<s_{i}<t} L_{i} g^{*}\left(h\left(s_{i}\right)\right) \\
& +C L \int_{s_{i}}^{t} \int_{s_{0}}^{s} g^{*}(u) \Delta u \Delta s+C L \int_{s_{i}}^{t} \int_{s_{0}}^{s} g^{*}(h(u)) \Delta u \Delta s .
\end{aligned}
$$

As, $g^{*}$ is increasing, therefore $g^{*}(h(t)) \leqslant g^{*}(t)$, further we can write

$$
g^{*}(t) \leqslant\left(m+C t_{f}-C s_{i}\right) \epsilon+\sum_{0<s_{i}<t} 2 L_{i} g^{*}\left(s_{i}\right)+2 C L \int_{s_{i}}^{t} \int_{s_{0}}^{s} g^{*}(u) \Delta u \Delta s .
$$

Using Lemma 3.7, we have

$$
g^{*}(t) \leqslant\left(m+C t_{f}-C s_{i}\right) \in \prod_{0<s_{i}<t}\left(1+2 L_{i}\right) e_{q}\left(t, s_{i}\right),
$$

where $\mathrm{q}=2 \mathrm{CL} \int_{s_{0}}^{s} \Delta \mathrm{u}$. If we determine $\mathrm{g}=\|\phi-w\|$, then $\mathrm{g}(\mathrm{t}) \leqslant(\mathrm{Tg})(\mathrm{t})$, which follows by utilizing abstract Grönwall lemma that $g(t) \leqslant g^{*}$, hence

$$
\|\phi(t)-\omega(t)\| \leqslant\left(m+C t_{f}-C s_{i}\right) \epsilon \prod_{0<s_{i}<t}\left(1+2 L_{i}\right) e_{q}\left(t, s_{i}\right) .
$$

Similarly we can establish the Hyers-Ulam-Rassias stability of (1.2) on J. Its proof will be omitted.

Theorem 5.3. If conditions $\left(\mathrm{A}_{1}\right)-\left(\mathrm{A}_{4}\right)$ hold, then, equation (1.2) has Hyers-Ulam-Rassias stability on J.

Example 5.4. Consider the following semilinear Volterra integro-dynamic equation:

$$
\omega^{\Delta}(t)=(t-2) \omega(t)+\int_{t_{0}}^{t} e_{p}(t, \omega(s)) \Delta s, \omega(0)=1, \quad t \in[0,3]_{T_{S^{\prime}}}
$$

and its associated inequality

$$
\left|\phi^{\Delta}(\mathrm{t})-(\mathrm{t}-2) \phi(\mathrm{t})-\int_{\mathrm{t}_{0}}^{\mathrm{t}} e_{\mathrm{p}}(\mathrm{t}, \phi(\mathrm{s})) \Delta s\right| \leqslant 1.5, \mathrm{t} \in[0,3]_{\mathrm{T}_{\mathrm{S}}} .
$$


Setting $p(t)=(t-2)$ and $K(t, s, w(s))=e_{p}(t, w(s))=e_{p}(t, s) e_{p}(s, w(s))$ for $t \in T_{S}$ and put $\epsilon=1.5$. If $\phi \in \mathrm{P}_{C}^{1}\left([0,3]_{T_{S}}, R\right)$ satisfies the inequality (5.3), then there exists $f \in P_{C}^{1}\left([0,3]_{T_{S}}, R\right)$ such that $|f(t)| \leqslant 1.5$ for $t \in T_{S}$. So we have

$$
\phi^{\Delta}(\mathrm{t})=(\mathrm{t}-2) \phi(\mathrm{t})+\int_{\mathrm{t}_{0}}^{\mathrm{t}} e_{\mathrm{p}}(\mathrm{t}, \phi(\mathrm{s})) \Delta \mathrm{s}+\mathrm{f}(\mathrm{t}), \quad \mathrm{t} \in \mathrm{T}_{\mathrm{S}},
$$

and the solution of Eq. (5.2) is given as

$$
\omega(t)=e_{p}(t, 0)+\int_{0}^{t} e_{p}(t, \Theta(s)) \int_{0}^{s} e_{p}(s, \omega(u)) \Delta u \Delta s .
$$

Based on our theoretical results, Eq. (5.2) has a unique solution in $P_{C}^{1}\left([0,3]_{T_{S}}, R\right)$ and is Hyers-Ulam stable on $[0,3]_{\mathrm{T}_{\mathrm{S}}}$.

\section{Conclusion}

This manuscript is about the establishment of Hyers-Ulam stability and Hyers-Ulam-Rassias stability of (1.1) and (1.2) with the utilization of fixed point approach. Furthermore, abstract Grönwall lemma, Lemma 3.6, and Lemma 3.7 presented a fruitful outcome to our end. Our work assures the existence of an exact solutions of (1.1) and (1.2) near to approximate solution. We added an example to show the validity of our results. In fact, our results are significant when finding exact solution is quite difficult and hence are important in approximation theory etc.

\section{References}

[1] R. P. Agarwal, A. S. Awan, D. ÓRegan, A. Younus, Linear impulsive Volterra integro-dynamic system on time scales, Adv. Difference Equ., 2014 (2014), 17 pages. 1

[2] Z. Ali, A. Zada, K. Shah, Ulam stability to a toppled systems of nonlinear implicit fractional order boundary value problem, Bound. Value Probl., 2018 (2018), 16 pages. 1

[3] Z. Ali, A. Zada, K. Shah, Ulam stability results for the solutions of nonlinear implicit fractional order differential equations, Hacettepe J. Math. Stat., 2018 (2018), (in press). 1

[4] C. Alsina, R. Ger, On some inequalities and stability results related to the exponential function, J. Inequal. Appl., 2 (1998), 373-380. 1

[5] S. András, A. R. Mészáros, Ulam-Hyers stability of dynamic equations on time scales via Picard operators, Appl. Math. Comput., 209 (2013), 4853-4864. 1

[6] D. D. Bainov, A. B. Dishliev, Population dynamics control in regard to minimizing the time necessary for the regeneration of a biomass taken away from the population, C. R. Acad. Bulgare Sci., 42 (1989), 29-32. 1

[7] D. D. Bainov, P. S. Simenov, Systems with impulse effect stability theory and applications, Ellis Horwood Limited, Chichester, (1989). 1

[8] M. Bohner, A. Peterson, Dynamic equations on time scales: an introduction with applications, Birkhäuser, Boston, (2001). 1, 3.6

[9] M. Bohner, A. Peterson, Advances in dynamics equations on time scales, Birkhäuser, Boston, (2003).

[10] J. J. DaChunha, Stability for time varying linear dynamic systems on time scales, J. Comput. Appl. Math., 176 (2005), 381-410.

[11] A. E. Hamza, K. M. Oraby, Stability of abstract dynamic equations on time scales, Adv. Difference Equ., 2012 (2012), 15 pages. 1

[12] S. Hilger, Analysis on measure chains-A unified approach to continuous and discrete calculus, Result math., 18 (1990), 18-56. 1

[13] D. H. Hyers, On the stability of the linear functional equation, Proc. Nat. Acad. Sci. U.S.A., 27 (1941), 222-224. 1

[14] S.-M. Jung, Hyers-Ulam stability of linear differential equations of first order, Appl. Math. Lett., 17 (2004), 1135-1140. 1

[15] S.-M. Jung, Hyers-Ulam-Rassias stability of functional equations in nonlinear analysis, Springer, New York, (2011). 1

[16] Y. J. Li, Y. Shen, Hyers-Ulam stability of linear differential equations of second order, Appl. Math. Lett., 23 (2010), 306-309. 1

[17] T. X. Li, A. Zada, Connections between Hyers-Ulam stability and uniform exponential stability of discrete evolution families of bounded linear operators over Banach spaces, Adv. Difference Equ., 2016 (2016), 8 pages.

[18] T. X. Li, A. Zada, S. Faisal, Hyers-Ulam stability of nth order linear differential equations, J. Nonlinear Sci. Appl., 9 (2016), 2070-2075. 1 
[19] Z. Lin, W. Wei, J. R. Wang, Existence and stability results for impulsive integro-differential equations, Facta Univ. Ser. Math. Inform., 29 (2014), 119-130. 1

[20] V. Lupulescu, A. Zada, Linear impulsive dynamic systems on time scales, Electron. J. Qual. Theory Differ. Equ., 2010 (2010), 30 pages. $1,3.7$

[21] S. I. Nenov, Impulsive controllability and optimization problems in population dynamics, Nonlinear Anal., 36 (1999), 881-890. 1

[22] M. Obłoza, Hyers stability of the linear differential equation, Rocznik Nauk.-Dydakt. Prace Mat., 13 (1993), 259-270. 1

[23] M. Obłoza, Connections between Hyers and Lyapunov stability of the ordinary differential equations, Rocznik Nauk.Dydakt. Prace Mat., 14 (1997), 141-146. 1

[24] C. Pötzsche, S. Siegmund, F. Wirth, A spectral characterization of exponential stability for linear time-invariant systems on time scales, Discrete Contin. Dyn. Syst., 9 (2003), 1223-1241. 1

[25] T. M. Rassias, On the stability of linear mappings in Banach spaces, Proc. Amer. Math. Soc., 72 (1978), 297-300. 1

[26] I. A. Rus, Grönwall lemmas: ten open problems, Sci. Math. Jpn., 70 (2009), 221-228. 3.8

[27] S. O. Shah, A. Zada, Uniform exponential stability for time varying linear dynamic systems over time scales, J. Anal. Number Theory, 5 (2017), 115-118. 1

[28] R. Shah, A. Zada, A fixed point approach to the stability of a nonlinear volterra integrodifferential equations with delay, Hacet. J. Math. Stat., 47 (2018), 615-623. 1

[29] S. O. Shah, A. Zada, Connections between Ulam-Hyers stability and uniform exponential stability of time varying linear dynamic systems over time scales, Sohag J. Math., 6 (2019), 1-4. 1

[30] S. O. Shah, A. Zada, Hyers-Ulam stability of non-linear Volterra integro-delay dynamic system with fractional integrable impulses on time scales, IJMSI., (in press). 1

[31] S. O. Shah, A. Zada, A. E. Hamza, Stability analysis of the first order non-linear impulsive time varying delay dynamic system on time scales, Qual. Theory Dyn. Syst., 2019 (2019), 16 pages. 1

[32] S. H. Tang, A. Zada, S. Faisal, M. M. A. El-Sheikh, T. X. Li, Stability of higher-order nonlinear impulsive differential equations, J. Nonlinear Sci. Appl., 9 (2016), 4713-4721. 1

[33] S. M. Ulam, A Collection of Mathematical Problems, Interscience Publisheres, New York-London, (1960). 1

[34] S. M. Ulam, Problems in modern mathematics, Science Editions John Wiley \& Sons, New York, (1964). 1

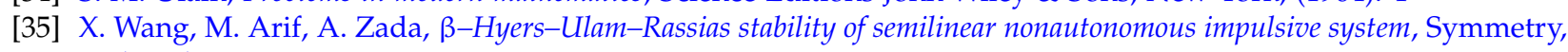
11 (2019), 18 pages. 1

[36] J. R. Wang, M. Fečkan, Y. Zhou, Ulam's type stability of impulsive ordinary differential equations, J. Math. Anal. Appl., 395 (2012), 258-264. 1

[37] J. R. Wang, M. Fečkan, Y. Zhou, On the stability of first order impulsive evolution equations, Opuscula Math., 34 (2014), 639-657. 1

[38] J. R. Wang, A. Zada, W. Ali, Ulam's-type stability of first-order impulsive differential equations with variable delay in quasi-Banach spaces, Int. J. Nonlinear Sci. Numer. Simul., 19 (2018), 553-560. 1

[39] J. R. Wang, Y. R. Zhang, A class of nonlinear differential equations with fractional integrable impulses, Commun. Nonlinear Sci. Numer. Simul., 19 (2014), 3001-3010. 1

[40] A. Younus, D. O’Regan, N. Yasmin, S. Mirza, Stability criteria for nonlinear volterra integro-dynamic systems, Appl. Math. Inf. Sci., 11 (2017), 1509-1517. 1

[41] B. Zada, Uniform exponential stability in the sense of Hyers and Ulam for periodic time varying linear systems, Differ. Equ. Appl., 10 (2018), 227-234. 1

[42] A. Zada, S. Ali, Stability analysis of multi-point boundary value problem for sequential fractional differential equations with non-instantaneous impulses, Int. J. Nonlinear Sci. Numer. Simul., 19 (2018), 763-774.

[43] A. Zada, W. Ali, S. Farina, Hyers-Ulam stability of nonlinear differential equations with fractional integrable impulses, Math. Methods Appl. Sci., 40 (2017), 5502-5514. 1

[44] A. Zada, S. Ali, Y. J. Li, Ulam-type stability for a class of implicit fractional differential equations with non-instantaneous integral impulses and boundary condition, Adv. Difference Equ., 2017 (2017), 26 pages.

[45] A. Zada, W. Ali, C. Park, Ulam's type stability of higher order nonlinear delay differential equations via integral inequality of Grönwall-Bellman-Bihari's type, Appl. Math. Comput., 350 (2019), 60-65.

[46] A. Zada, U. Riaz, F. Ullah Khan, Hyers-Ulam stability of impulsive integral equations, Boll. Unione Mat. Ital., 2018 (2018), 1-15.

[47] A. Zada, S. O. Shah, Hyers-Ulam stability of first-order non-linear delay differential equations with fractional integrable impulses, Hacettepe J. Math. Stat., 47 (2018), 1196-1205. 1

[48] A. Zada, S. O. Shah, S. Ismail, T. X. Li, Hyers-Ulam stability in terms of dichotomy of first order linear dynamic systems, Punjab Univ. J. Math. (Lahore), 49 (2017), 37-47. 1

[49] A. Zada, S. O. Shah, Y. J. Li, Hyers-Ulam stability of nonlinear impulsive Volterra integro-delay dynamic system on time scales, J. Nonlinear Sci. Appl., 10 (2017), 5701-5711. 1

[50] A. Zada, O. Shah, R. Shah, Hyers-Ulam stability of non-autonomous systems in terms of boundedness of Cauchy problems, Appl. Math. Comput., 271 (2015), 512-518.

[51] A. Zada, S. Shaleena, T. X. Li, Stability analysis of higher order nonlinear differential equations in $\beta$-normed spaces, Math. Methods Appl. Sci., 42 (2019), 1151-1166. 
[52] A. Zada, F. Ullah Khan, U. Riaz, T. X. Li, Hyers-Ulam stability of linear summation equations, Punjab Univ. J. Math. (Lahore), 49 (2017), 19-24.

[53] A. Zada, P. U. Wang, D. Lassoued, T. X. Li, Connections between Hyers-Ulam stability and uniform exponential stability of 2-periodic linear nonautonomous systems, Adv. Difference Equ., 2017 (2017), 7 pages.

[54] A. Zada, M. Yar, T. X. Li, Existence and stability analysis of nonlinear sequential coupled system of Caputo fractional differential equations with integral boundary conditions, Ann. Univ. Paedagog. Crac. Stud. Math., 17 (2018), $103-125$. 1 